\title{
OMAE2007-29294
}

\section{NUMERICAL SIMULATION OF SLOSHING IN LNG TANKS WITH A COMPRESSIBLE TWO-PHASE MODEL}

\author{
Rik Wemmenhove \\ Department of Mathematics \\ University of Groningen \\ PO Box 800, 9700 AV Groningen \\ The Netherlands \\ Email: r.wemmenhove@math.rug.nl \\ Arthur E.P. Veldman \\ Department of Mathematics \\ University of Groningen \\ PO Box 800, 9700 AV Groningen \\ The Netherlands
}

\author{
Roel Luppes \\ Department of Mathematics \\ University of Groningen \\ PO Box 800, 9700 AV Groningen \\ The Netherlands
}

\begin{abstract}
The study of liquid dynamics in LNG tanks is getting more and more important with the actual trend of $L N G$ tankers sailing with partially filled tanks. The effect of sloshing liquid in the tanks on pressure levels at the tank walls and on the overall ship motion indicates the relevance of an accurate simulation of the fluid behaviour. This paper presents the simulation of sloshing $L N G$ by a compressible two-phase model and the validation of the numerical model on model-scale sloshing experiments.
\end{abstract}

The details of the numerical model, an improved Volume Of Fluid (iVOF) method, are presented in the paper. The program has been developed initially to study the sloshing of liquid fuel in spacecraft. The micro-gravity environment requires a very accurate and robust description of the free surface. Later, the numerical model has been used for calculations for different offshore applications, including green water loading.

The model has been extended to take two-phase flow effects into account. These effects are particularly important for sloshing in tanks. The complex mixture of the liquid and gas phase around the free surface imposes a challenge to numerical simulation. The two-phase flow effects (air entrapment and entrainment) are strongly affected by both the filling ratio of the tank and the irregular motion of the tank in typical offshore conditions.

The velocity field and pressure distribution around the interface of air and LNG, being continuous across the free surface, requires special attention. By using a newly-developed gravityconsistent discretisation, spurious velocities at the free surface are prevented. The equation of state applied in the compressible cells in the flow domain induces the need to keep track on the pressure distribution in both phases, as the gas density is directly coupled to the gas pressure.

The numerical model is validated on a 1:10 model-scale sloshing model experiment. The paper shows the results of this validation for different filling ratios and for different types of motion of the sloshing tank. 


\section{NOMENCLATURE}

$\begin{array}{lll}\mathrm{dt} & {[s]} & \text { time step } \\ \mathrm{F} & {[\mathrm{N}]} & \text { body force } \\ F_{b} & {[-]} & \text { open cell fraction } \\ F_{S} & {[-]} & \text { liquid cell fraction } \\ \mathrm{g} & {\left[\mathrm{ms}^{-2}\right]} & \text { gravity vector } \\ \mathrm{h} & {[\mathrm{m}]} & \text { grid cell spacing } \\ \mathrm{n} & {[-]} & \text { time level } \\ \mathrm{p} & {[\mathrm{Pa}]} & \text { pressure } \\ \mathrm{u} & {\left[\mathrm{ms}^{-1}\right]} & \text { velocity } \\ \mathrm{V} & {\left[\mathrm{m}^{3}\right]} & \text { volume } \\ \gamma & {[-]} & \text { adiabatic coefficient } \\ \mu & {\left[\mathrm{kgm}^{-1} \mathrm{~s}^{-1}\right]} & \text { dynamic viscosity } \\ \rho & {\left[\mathrm{kgm}^{-3}\right]} & \text { density }\end{array}$

\section{INTRODUCTION}

During all types of weather conditions, partially filled LNG tanks are facing varying pressure loads on their walls. Especially during more violent weather conditions, the interaction between ship movement and the movement of LNG inside tanks is worthwile to investigate. The fluid distribution in the tanks strongly depends on both the tank filling ratio and the weather conditions the tanker is subjected to. Inside the tanks, the fluid is generally a complex mixture of different fluids, with a strong mixing for violent flow conditions.

The simulation of the fluid behaviour in LNG tanks shows an analogy with the simulation of hydrodynamic wave loading on other offshore structures, so similar models can be used. Most models focus on specific aspects of free-surface flows, such as wave impact of aerated flows on walls or the velocity field under breaking waves.

As soon as flow conditions are getting more violent, using a two-phase flow model is strongly recommendable. However, the small spatial and temporal scales of entrapped and entrained air in the flow are a serious problem (see figure 1).

Existing two-phase models mainly focus mainly on single bubbles or on quite regular waves. Scardovelli and Zaleski [5] give a nice overview of existing two-phase flow models. During the last few years, there is much progress in the simulation of two-phase flows [1], [4], [8]. Furthermore, computer power is getting cheaper, increasing the possibilities to simulate large problems. Keeping track of the dynamics of the air phase in sloshing experiments is difficult. The required resolution in combination with the long time series induces the need to use powerful computers, but even then long calculation times remain an issue.

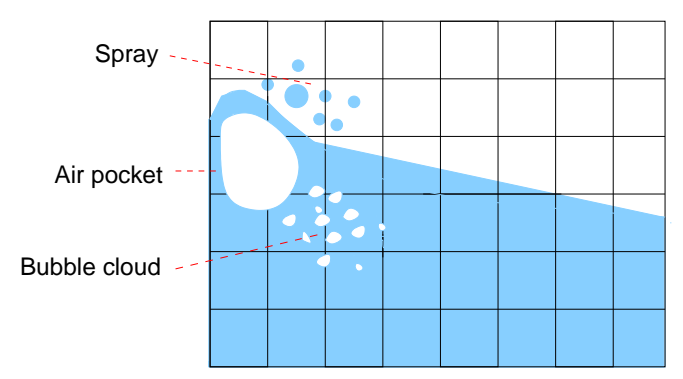

Figure 1. Two-phase phenomena at a breaking wave. Air pockets are difficult to 'catch' within the grid, bubbles and spray are almost impossible to track as individual entities.

The present paper shows the results of two-phase numerical simulations of sloshing in a tank. The simulations are validated on a series of 1:10 scale sloshing model experiments. In comparison with earlier sloshing model experiments [7], the current validation experiments are on a larger model scale. Moreover, the numerical model is able to use the experimental global motion time-traces from the sloshing tank as input for the simulations.

In the present paper the two-phase numerical model is presented. The model is aimed to simulate a wide range of offshore problems, but in this paper only the sloshing application is described.

The first section of this paper shows the governing equations the model is based on. After that, the main aspects of the discretisation of the numerical model are shown. Special attention is paid to the compressibility of the compressible phase and to the proper calculation of the density. In the last section the results of the validation of the numerical model on the sloshing experiments are shown.

\section{GOVERNING EQUATIONS}

For each point in the domain the fluid motion is governed by the continuity equation and the momentum equation. Both equations are aggregated, i.e. one set of equations is used to describe the behaviour of the two different fluids together.

$$
\begin{array}{r}
\frac{\partial \rho}{\partial t}+\nabla \cdot(\rho \mathbf{u})=0 \\
\frac{\partial(\rho \mathbf{u})}{\partial t}+\underbrace{\nabla \cdot(\rho \mathbf{u u})}_{\text {convection }}+\nabla p-\underbrace{\nabla \cdot(\mu \nabla \mathbf{u})}_{\text {diffusion }}-\rho \mathbf{F}=0
\end{array}
$$

with an external body force $\mathbf{F}$, velocity $\mathbf{u}$, pressure $p$, density $\rho$ and dynamical viscosity $\mu$.

For incompressible flow conditions the velocity field is 
divergence-free, reducing the continuity equation to

$$
\nabla \cdot \mathbf{u}=0
$$

The continuity equation and momentum equation are, after semidiscretisation in time, combined to the pressure Poisson equation to compute the new pressure value and velocity field. This pressure equation is shown in the next section.

\section{NUMERICAL MODEL iVOF algorithm}

The advection of water and air in the current model is based on the Volume Of Fluid (VOF) algorithm as developed by Hirt and Nichols [3].

As long as a one-phase approach is used for the model, the incompressible Navier-Stokes equations are solved with a free-surface boundary condition on the free boundary. The VOF function $F_{s}$ (with values between 0 and 1) determines whether or not the flow field in a grid cell is calculated.

This is in contrast with the two-phase approach, where the liquid-gas interface is no longer considered as a free surface, although the interface is still reconstructed using the VOF algorithm. Numerically important is now the density jump across the liquid-gas interface, as the density can increase or decrease a factor 1000 , imposing a challenge to the numerical stability of the model. The liquid fraction $F_{S}$ is now used as well to calculate the aggregated density in a grid cell.

The numerical model has been implemented in a 3D VOF Navier-Stokes solver called CoMFLOW. Compared with the original VOF algorithm [3], a local height function improves the treatment of the free surface. The program has been developed initially by the University of Groningen to study the sloshing of liquid fuel in spacecraft [2], [6]. The micro-gravity environment requires a very accurate and robust description of the free surface. In close cooperation with MARIN (Maritime Research Institute Netherlands), this methodology was later extended to the calculation of green water loading on a fixed bow deck. Also anti-roll tanks, including the coupling with ship motions, were investigated. Furthermore, the entry of a wedge in a fluid was studied as part of the RuG-MARIN cooperation, as well as the wave impact loads on fixed structures [4].

\section{Cell labeling}

Before describing the discretisation of the equations, it is worthwhile to describe the cell labeling first. The variable $F_{b}$ describes the fraction of a grid cell open for fluid, while the variable $F_{s}$ describes the fraction of a grid cell filled with the liquid phase. The Navier-Stokes equations are solved in grid cells containing at least one of the fluids.
Every grid cell is given a label to distinguish between boundary, air and fluid. Two classes of labeling exist: geometry cell labels and fluid cell labels.

The geometry labeling at each time step divides the cells into three classes:

F(low) cells: All cells with $F_{b} \geq 0$

$\mathbf{B}$ (oundary) cells: All cells adjacent to a F-cell

(e) $\mathbf{X}$ (ternal) cells: All remaining cells

The free-surface cell labeling is a subdivision of the $\mathbf{F}$ cells, consisting of 3 subclasses:

E(mpty) cells: All cells with $F_{s}=0$

S(urface) cells: All cells adjacent to an E-cell

F'(luid) cells: All remaining F-cells
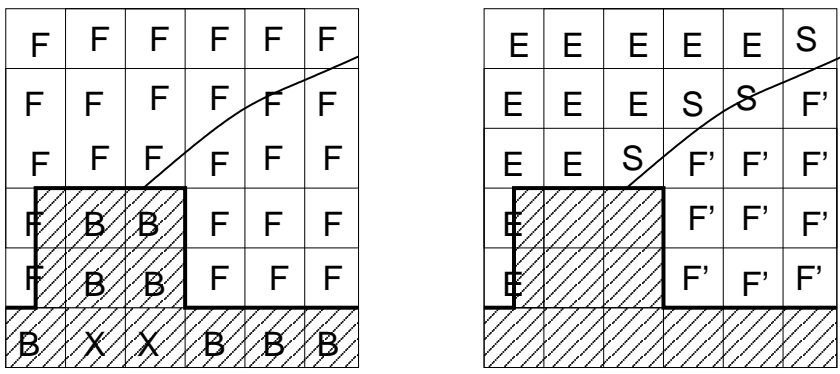

Figure 2. Geometry cell labeling (left) and free-surface cell labeling for wave impact on a rectangular container

Figure 2 shows an example of geometry cell labeling and free-surface cell labeling for wave impact from the right on a rectangular container.

The labeling system is retained in the two-phase model. In the one-phase model the empty cells were truly empty, as no computations were performed there, while boundary conditions for the free surface were prescribed around surface cells. In the two-phase model, however, the empty cells contain the fluid with the smallest density. The Navier-Stokes equations are also solved in these cells.

\section{Discretisation}

The discretisation of the Navier-Stokes equations is done on a staggered Cartesian grid, which means that the pressure is set in the cell centers and the velocity components in the middle of the cell faces between two grid cells, see figure 3. Spatial discretisation is done by means of a first-order upwind scheme. For compressible flow conditions, the Navier-Stokes equations, 


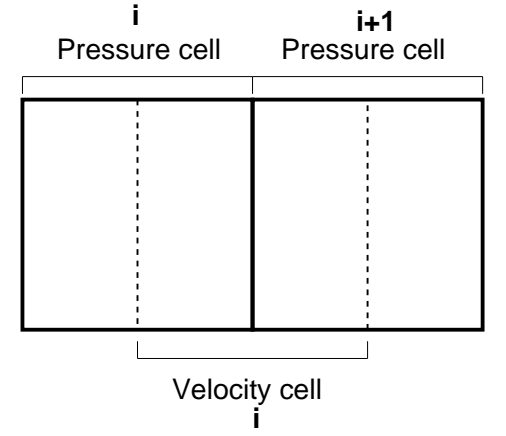

Figure 3. Staggered Cartesian grid; pressures and densities in cell centers and velocities at cell faces. The continuity equation is calculated in pressure cells, the momentum equation in velocity cells.

as given by eq.(1) and eq.(2), are discretised in time according to the explicit first order Forward Euler method. The momentum equation is divided by the density first, giving

$$
\begin{gathered}
\frac{\rho^{n+1}-\rho^{n}}{d t}+\rho^{n} \nabla \mathbf{u}^{n+1}+\mathbf{u}^{n} \nabla \rho^{n}=0 \\
\frac{\mathbf{u}^{n+1}-\mathbf{u}^{n}}{d t}+\frac{\mathbf{u}^{n}}{\rho^{n}} \frac{\rho^{n+1}-\rho^{n}}{d t}+\frac{1}{\rho^{n}} \nabla \cdot(\rho \mathbf{u u})^{n} \\
+\frac{1}{\rho^{n}} \nabla p^{n+1}-\frac{1}{\rho^{n}} \nabla\left(\mu^{n} \nabla \mathbf{u}^{n}\right)-\mathbf{F}^{n}=0
\end{gathered}
$$

with $n$ the old time level and $n+1$ the new time level.

\section{Towards the pressure Poisson equation}

A few more steps are needed to formulate the pressure Poisson equation for compressible flow. The first step is to take the divergence of the momentum equation. The term $\nabla \cdot \mathbf{u}^{n+1}$ is isolated and combined with the momentum equation. This results in the pressure Poisson equation:

$$
\begin{array}{r}
d t \nabla \cdot\left(\frac{1}{\rho^{n}} \nabla p^{n+1}\right)=\frac{1}{\rho^{n}} \frac{\rho^{n+1}-\rho^{n}}{d t}+\frac{\mathbf{u}^{n}}{\rho^{n}} \nabla \rho^{n}+\nabla \cdot \mathbf{u}^{n} \\
+\nabla\left(\frac{\mathbf{u}^{n}}{\rho^{n}}\left(\rho^{n}-\rho^{n+1}\right)\right)-d t \nabla\left(\frac{1}{\rho^{n}} \nabla\left(\rho^{n} \mathbf{u}^{n} \mathbf{u}^{n}\right)\right) \\
+d t \nabla\left(\frac{1}{\rho^{n}} \nabla\left(\mu^{n} \nabla \mathbf{u}^{n}\right)\right)+d t \nabla \mathbf{F}^{n}
\end{array}
$$

In the compressible Poisson equation (6), the pressure equation can also be formulated as

$$
d t \nabla \cdot\left(\frac{1}{\rho^{n}} \nabla p^{n+1}\right)=\frac{1}{\rho^{n}} \frac{\rho^{n+1}-\rho^{n}}{d t}+\frac{\mathbf{u}^{n}}{\rho^{n}} \nabla \rho^{n}
$$

$$
+\nabla\left(\frac{\mathbf{u}^{n}}{\rho^{n}}\left(\rho^{n}-\rho^{n+1}\right)\right)+\nabla \cdot \tilde{\mathbf{u}}^{n}
$$

with $\tilde{\mathbf{u}}^{n}$ the intermediate velocity following from the convective, diffusive and force terms:

$$
\tilde{\mathbf{u}}^{n}=\mathbf{u}^{n}-d t\left(\frac{1}{\rho^{n}} \nabla\left(\rho^{n} \mathbf{u}^{n} \mathbf{u}^{n}\right)\right)+d t\left(\frac{1}{\rho^{n}} \nabla\left(\mu^{n} \nabla \mathbf{u}^{n}\right)\right)+d t \mathbf{F}^{n}
$$

The 'compressible' terms in the Poisson equation are numerically dangerous because of the spatial and temporal derivatives of the density. Around the free surface, the density can jump from 1 to 1000. The first and the second term of the RHS of eq.(7) can be recognized as the $\frac{D \rho}{D t}$ term, which should be equal to zero for incompressible flow. We can split the density in an incompressible liquid part $\rho_{l}$ and a compressible gas part $\rho_{g}$. Using the VOF function $F_{s}$, the cell density $\rho$ is given by $\frac{F_{s}}{F_{b}} \rho_{l}+\frac{\left(F_{b}-F_{s}\right)}{F_{b}} \rho_{g}$, or $\tilde{F}_{s} \rho_{l}+\left(1-\tilde{F}_{s}\right) \rho_{g}$. Writing out the term $\frac{\rho^{n+1}-\rho^{n}}{d t}+u^{n} \nabla \rho^{n}$ for a mass cell gives

$$
\begin{array}{r}
\frac{\partial \rho}{\partial t}=\frac{d\left(\tilde{F}_{s} \rho_{l}+\left(1-\tilde{F}_{s}\right) \rho_{g}\right)}{d t}=\frac{d}{d t}\left(\tilde{F}_{s} \rho_{l}\right)+\frac{d}{d t}\left(\left(1-\tilde{F}_{s}\right) \rho_{g}\right) \\
=\rho_{l} \frac{d \tilde{F}_{s}}{d t}+\underbrace{\tilde{F}_{s} \frac{d}{d t}\left(\rho_{l}\right)}_{=0}+\rho_{g} \frac{d}{d t}\left(1-\tilde{F}_{s}\right)+\left(1-\tilde{F}_{s}\right) \frac{d}{d t} \rho_{g} \\
=\left(\rho_{l}-\rho_{g}\right) \frac{d \tilde{F}_{s}}{d t}+\left(1-\tilde{F}_{s}\right) \frac{\partial\left(\rho_{g}\right)}{\partial t}
\end{array}
$$

and

$$
\begin{array}{r}
u \nabla \rho=u \frac{d\left(\tilde{F}_{s} \rho_{l}+\tilde{F}_{s} \rho_{g}\right)}{d x} \\
=u \rho_{l} \frac{d \tilde{F}_{s}}{d x}+\underbrace{u \tilde{F}_{s} \frac{\partial \rho_{l}}{\partial x}}_{=0}+u\left(1-\tilde{F}_{s}\right) \frac{\partial \rho_{g}}{\partial x}+u \rho_{g} \frac{\partial\left(1-\tilde{F}_{s}\right)}{\partial x}
\end{array}
$$

Adding the two terms gives

$$
\left(\rho_{l}-\rho_{g}\right) \underbrace{\left.\frac{\partial \tilde{F}_{s}}{\partial t}+u \frac{\partial \tilde{F}_{s}}{\partial x}\right)}_{=0}+\left(1-\tilde{F}_{s}\right)\left(\frac{\partial \rho_{g}}{\partial t}+u \frac{\partial \rho_{g}}{\partial x}\right)
$$

By the split-up of the density into a liquid and gas part, the pressure Poisson equation (7) reduces to

$$
\begin{gathered}
d t \nabla \cdot\left(\frac{1}{\rho^{n}} \nabla p^{n+1}\right)=\frac{\left(1-\tilde{F}_{s}\right)}{\rho^{n}} \frac{\rho_{g}^{n+1}-\rho_{g}^{n}}{d t} \\
+\frac{\left(1-\tilde{F}_{S}\right) \mathbf{u}^{n}}{\rho^{n}} \nabla \rho_{g}^{n}+\nabla\left(\frac{\mathbf{u}^{n}}{\rho^{n}}\left(\rho^{n}-\rho^{n+1}\right)\right)+\nabla \cdot \tilde{\mathbf{u}}^{n}
\end{gathered}
$$




\begin{tabular}{clll}
\hline Flow model & 2-ph com & 2-ph inc & 1-ph inc \\
\hline Liquid cell & $\rho=\rho_{l}=1000$ & $\rho=\rho_{l}=1000$ & $\rho=1000$ \\
Gas cell & $\rho=\rho_{g}=f(p)$ & $\rho=\rho_{g}=1$ & $\rho=0$ \\
Mixed cell & $\rho=f\left(F_{s}, p\right)$ & $\rho=f\left(F_{s}\right)$ & $\rho=f\left(F_{s}\right)$ \\
\hline
\end{tabular}

Table 1. Density for different cell categories and models

The remaining derivatives of the gas densities do not contain large jumps, as these derivatives are only determined by the compression and expansion of the gas phase. In eq.(12), different terms in the RHS require information of the density on the new time level. To start with the first term, the gas density can be substituted using the adiabatic relationship:

$$
\frac{\rho_{g}^{n+1}}{\rho_{g, 0}}=\left(\frac{p_{g}^{n+1}}{p_{g, 0}}\right)^{\frac{1}{\gamma}}
$$

with $p_{g, 0}$ and $\rho_{g, 0}$ the reference gas pressure and gas density values. The adiabatic coefficient $\gamma$ has a value of 1.4 for pure air. The atmospheric pressure and ambient density values are used for $p_{g, 0}$ and $\rho_{g, 0}$, this is a choice that has been made. Another option could be to use the pressure and density values from the previous time step, but that approach may lead to the propagation of small numerical errors.

Regarding eq.(13), it is important to keep track on a positive density value, as negative density values do not have a physical meaning. This implies that the pressure needs to be positive as well, a condition that needs to be fulfilled during the pressure iteration.

\section{Density treatment}

Special attention should be given to the discretisation of the density. Given the staggered arrangement of grid variables, see figure 3, pressure and density are both located in the cell centers.

For compressible two-phase flow, the density in the cell center is calculated as

$$
\rho=\frac{F_{s}}{F_{b}} \rho_{l}+\frac{\left(F_{b}-F_{s}\right)}{F_{b}} \rho_{g},
$$

so in fact the density is a function of the liquid cell fraction $F_{S}$ and pressure $p$ (see table). The density is coupled to the pressure value in the cell centers, however the staggered variable arrangement induces the need to calculate the densities at the cell edges as well, as will be shown below.
According to the solution method, the velocity field at the new time level is acquired by adding a term to the old velocity field (see equation (5)). Since the velocity field is highly continuous between the old and new time level, this term, $\nabla p / \rho$, should also be smooth. As the pressures are located in cell centers, the term $\nabla p$ has to be calculated in a control volume located between two cell centers. The density value $\rho$ should hold - and be defined properly - in the control volume which is located between the cell centers.

Consider the situation in figure 4 . The free surface dividing water (below) and air (above) is in this case a straight line with a slope of $\frac{1}{2}$. We need for both the horizontal and vertical direction a proper discretisation of the term $\nabla p / \rho$. The suggested control volumes for these terms are depicted with dashed lines, between cells A and F (vertical), and E and A (horizontal). As the pressure values are registrated at the circles, the discretization can be straighforward: $\left.\frac{1}{\rho} \frac{\partial p}{\partial x}\right|_{E A} \approx \frac{1}{h_{x}} \frac{\left(P_{A}-P_{E}\right)}{\rho_{E A}}$. Near the free surface, however, the density averaging is not clear beforehand. Consider for example the density $\rho_{A B}$ in the control volume between $A$ and $B$. Choices like 1 ("cell centers $A$ and $B$ both in air"), 125.875 ("average of complete cells $A$ and $B$ ") or even 63.4375 ("average of right half of cell $A$ and left half of cell $B$ ") can be defended.

Does it matter? In fact, quite a lot. Consider the situation above, and make it stationary by setting the gravity vector $g=$ $\left(g_{x}, g_{z}\right)^{T}=(5,-10)^{T}$. In that case, with all time derivatives and velocities zero, the momentum equations (2) are reduced to

$$
\frac{\partial p}{\partial x}=\rho_{x} g_{x}, \quad \frac{\partial p}{\partial z}=\rho_{z} g_{z}
$$

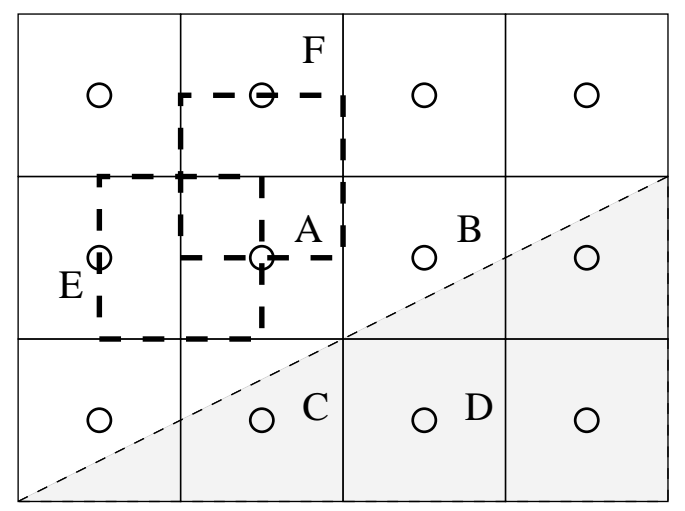

Figure 4. Averaging of density. The cells are numbered according to their position in the flow field.

Concentrating on the four cells $\mathrm{A}, \mathrm{B}, \mathrm{C}$ and $\mathrm{D}$, we take the simple approach of defining the densities between the cells as 
the average of the weighted cell averages. So cell averages are approximately $\rho_{A}=1, \quad \rho_{B}=250, \quad \rho_{C}=750$ and $\rho_{D}=1000$. Further we suppose the cells have unit size. Integrating the pressure in clockwise direction from A to D (via B) gives

$$
\begin{array}{r}
P_{D}=P_{B}-h_{z} g_{z} \rho_{B D}=P_{A}+h_{x} g_{x} \rho_{A B}-h_{z} g_{z} \rho_{B D} \\
=P_{A}+5 \cdot 125+10 \cdot 625=P_{A}+6875
\end{array}
$$

while integrating in counterclockwise direction (from $A$ to $D$ via C) gives

$$
\begin{array}{r}
P_{D}=P_{C}+h_{x} g_{x} \rho_{C D}=P_{A}-h_{z} g_{z} \rho_{A C}+h_{x} g_{x} \rho_{C D} \\
=P_{A}+10 \cdot 375+5 \cdot 875=P_{A}+8125 .
\end{array}
$$

This inconsistency (the contour integral not being zero) is immediately visible numerically: spurious velocities will occur around the free surface, see figure 5. So a more consistent density discretisation, which at least will cope correctly with stationary situations, is needed. It requires the gravitational force $\rho \mathbf{g}$ to be conservative. This very simple skewed-gravity example, however,
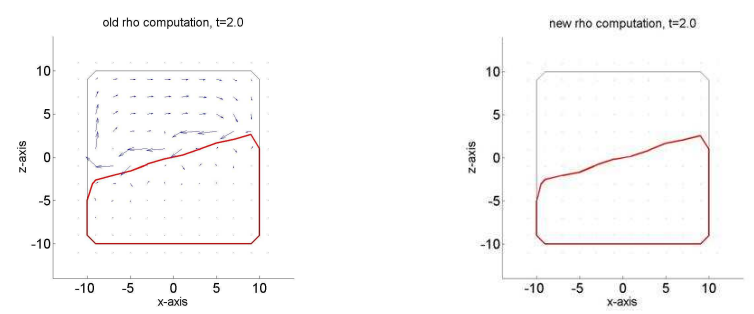

Figure 5. Spurious velocities due to simple averaging of density (left) disappear when a gravity-consistent density averaging is used (right).

provides a strategy for such a gravity-consistent discretisation. The pressure is known analytically: hydrostatic and 'aerostatic' in both phases, respectively. If we choose $p=0$ at the free surface, then (see figure 6): $P_{A}=-l_{1} \rho_{1}|\mathbf{g}|=d_{1} \cos \alpha \frac{g_{z}}{\cos \alpha}=d_{1} \rho_{1} g_{z}$ where $\alpha$ is the slope angle, and $P_{C}=l_{2} \rho_{2}|\mathbf{g}|=-d_{2} \rho_{2} g_{z}$. Together with $\frac{1}{\rho_{z}} \frac{\partial p}{\partial z}=g_{z}$, this gives

$$
\rho_{A C}=\frac{1}{g_{z}} \frac{P_{A}-P_{C}}{d_{1}+d_{2}}=\frac{d_{1} \rho_{1}+d_{2} \rho_{2}}{d_{1}+d_{2}} .
$$

A similar construction simply gives $\rho_{A B}=\rho_{1}$, because both cell centers are in the fluid with density $\rho_{1}$. Using the local height

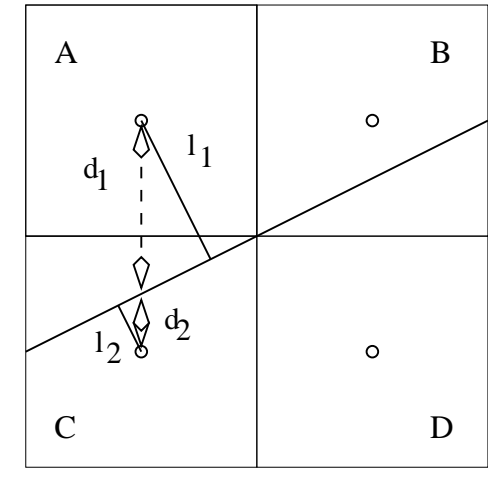

Figure 6. Gravity-consistent discretisation

function also used in the iVOF algorithm, the distances $d_{1}$ and $d_{2}$ can easily be computed. In the given example, this leads to $\rho_{A B}=1, \rho_{A C} \approx 250, \rho_{B D} \approx 750$ and $\rho_{C D}=1000$, which gives a consistent density field and no spurious velocities. Note that in this approach, the cell pressure was in some sense identified with the pressure in the center of the cell. This leads to the possibility of other ways of averaging the density, besides this 'cellcentered'-approach. Some of them are still under investigation.

\section{MODEL RESULTS: SLOSHING IN LNG TANKS}

In this section the validation of the numerical model on sloshing in LNG tanks is described. As mentioned in the Introduction, the fluid behaviour in partially-filled LNG tanks needs investigation. To study this sloshing behaviour, both numerical simulations and model scale experiments have been carried out. For two test cases with low and high tank filling ratios the numerical and experimental results will be described.

\section{Setup of the model tests}

The model tests on scale 1:10 have been carried out to generate validation material for the numerical model. To match simulation and experiment, the numerical simulations are carried out on the same scale as the model experiments. The experimental setup has been designed as a 2D cross-section of a sloshing tank. Prior to the tests at DNV (Det Norske Veritas) in Oslo, the entire test setup (except the oscillator to move the tank) has been built and verified at MARIN.

The sloshing tank model was based on an LNG tank inside a No. $96 \mathrm{LNG}$ carrier. The inner side of the tank (the part open to fluid) has a width of $3897 \mathrm{~mm}$ and a height of $2697 \mathrm{~mm}$ on model scale. The tank is filled with water, while the front side and back side are made of perspex to enable visualisation of the fluid motion inside the tank.

Figures 7 and 11 show screenshots of the sloshing tank for low and high filling ratios. As visible in these figures, the side walls 
of the tank are fitted with a number of measurement panels for pressure transducers, while the water height in the tank is measured by means of 12 water height probes. Furthermore, the tank is equipped with two cameras to monitor the fluid configuration. The global motion time-traces of the tank, which are measured during the experiments, are used as input for the simulations. The motion of the tank is considered as a moving coordinate frame in the simulations to ensure similar conditions for simulations and experiment. The origin of the coordinate system is chosen at the center of the tank bottom.

\section{Low filling ratio, sway motion}

The first test case is the case with a low tank filling ratio of 10 percent, while the tank is subject to a regular sway motion. The oscillation period of this test case is $11.0 \mathrm{~s}$. The sway motion of the experiment is used as input for the simulation.

Figure 7 shows the initial fluid configuration and the configuration at $t=15 \mathrm{~s}$. Although the amplitude of the sway motion is
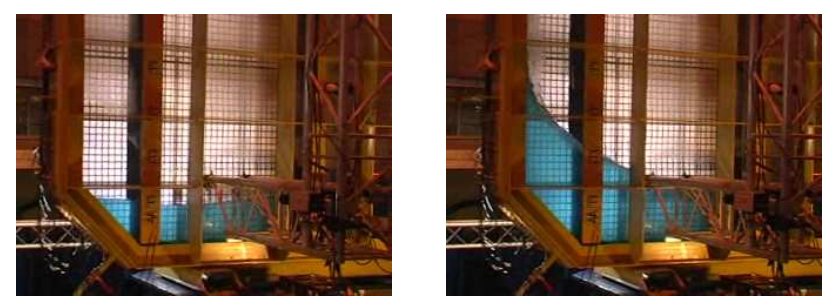

Figure 7. Screenshots of the 10 percent filling ratio sloshing model experiment. The left picture shows the fluid configuration at $t=0 \mathrm{~s}$, the right picture the configuration at $t=15 \mathrm{~s}$.

less than $10 \mathrm{~cm}$, a strong sloshing motion of the fluid is induced by the tank oscillation. In particular at the side walls, the water height strongly increases during the impact of the sloshing liquid, up to about one meter. Figure 8 shows the snapshots of the simulation, which are taken at the same moments in time as the screenshots in Figure 7.

The water height at the left wall during the 'impact' of the sloshing water seems to be lower in the simulation than in the experiment. To evaluate the water height development in time, Figure 9 shows the development of the water height at sensor 9, which is located about 1 meter from the left side wall. The agreement between simulations and experiment is good, however the two-phase simulation shows a smaller amplitude than both the one-phase simulation and the experiment. The development of the pressure level is important to predict the forcing on the tank walls due to the sloshing liquid. Figure 10 shows the pressure development for the sensor fitted at the lowest position of the right side wall, pressure transducer $1(x=1.656 m, z=0.152 m)$.
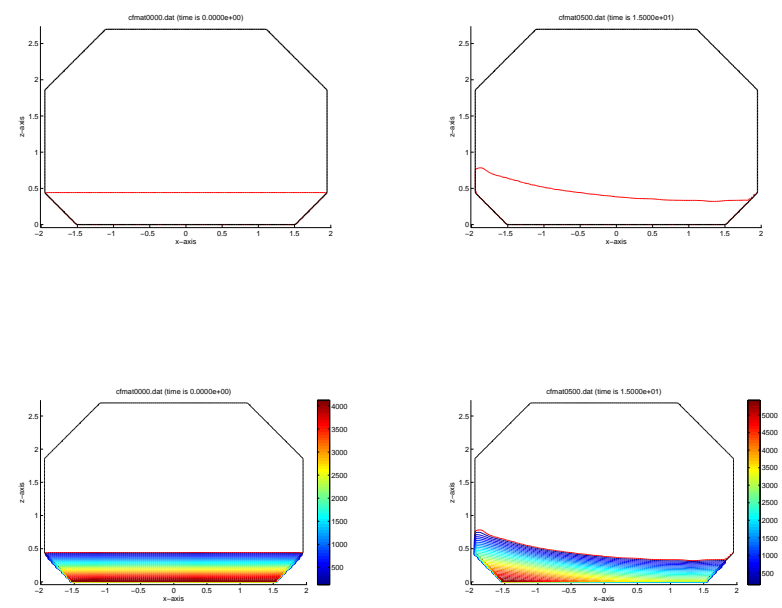

Figure 8. Snapshots of the 10 percent filling-ratio sloshing simulation with two-phase flow. Above the location of the free surface, below the pressure contours. The left picture shows the fluid configuration at $t=0 \mathrm{~s}$, the right picture the configuration at $t=15 \mathrm{~s}$. Note the absence of pressure contour lines in the air-filled part of the tank due to the small pressure gradient.

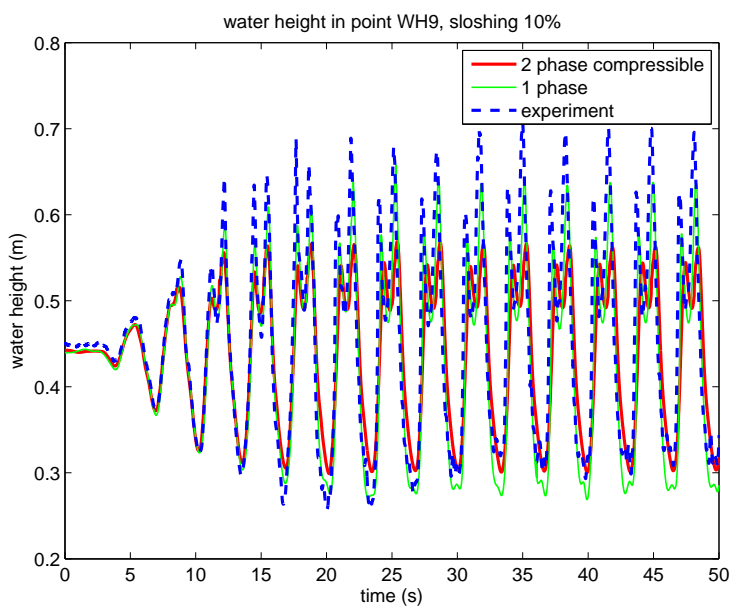

Figure 9. Water height development for 10 percent filling-ratio at sensor 9, about $1 \mathrm{~m}$ from the left side wall.

Regarding the water height development in Figure 9 and the pressure level development in Figure 10, the simulation shows the same patterns as for the experiment, but there are especially differences in water height and pressure level development near the side walls. The difference may have to do with the spatial and temporal scales for the experiment that are involved in the 


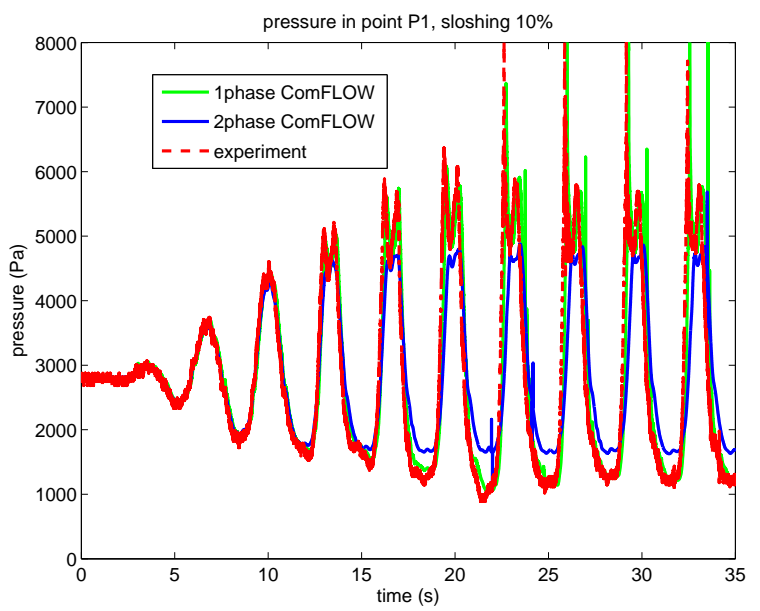

Figure 10. Pressure level development for 10 percent filling-ratio at pressure sensor 1, at the side wall.

impact of the sloshing water on the side walls of the tank. Only a thin liquid layer moves upwards along the side wall during the impact of the sloshing liquid, and the upward movement of this jet occurs very rapidly. The grid resolution in the simulation is quite coarse, $128 \times 128$. Increasing the resolution may improve the agreement with the water height and pressure level of the experiment, however computation times strongly increase for finer grids, especially for the long time series that are required for sloshing experiments. For the two-phase simulations, another issue to keep in mind is the first-order upwind spatial discretisation, which adds artificial viscosity to the fluid. Using a less dissipative second-order upwind discretisation may reduce the numerical damping of the sloshing liquid.

\section{High filling ratio, sway motion}

The second test case is the case with a high tank filling-ratio of 95 percent, while the tank is subjected to a regular sway motion. The oscillation period of the sway motion is now $8.3 \mathrm{~s}$. The sway motion of the experiment is used as input for the simulation.

Figure 11 shows the initial fluid configuration and the configuration at $t=8.8 \mathrm{~s}$.

In contrast with the low filling ratio test case, the tank is now almost completely filled with water. This means that only a small volume of air is present within the tank. The air in the top of the tank can be considered as entrapped, while air entrainment (the bubble clouds just below the free surface) is clearly visible as well. The air pocket is moving to the left and right part of the tank due to the sway motion. Figure 12 shows the snapshots of the simulation that are taken at the same time as the screenshots in Figure 11. At $t=8.8 \mathrm{~s}$ the air pocket is located at the left side
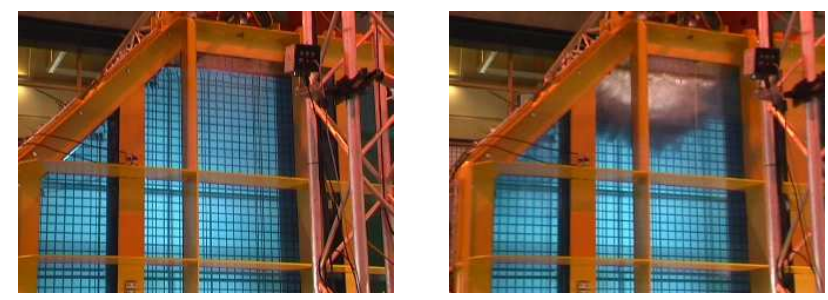

Figure 11. Screenshots of the 95 percent filling-ratio sloshing model experiment. The left picture shows the fluid configuration at $t=0 \mathrm{~s}$, the right picture the configuration at $t=8.8 \mathrm{~s}$.

of the tank.
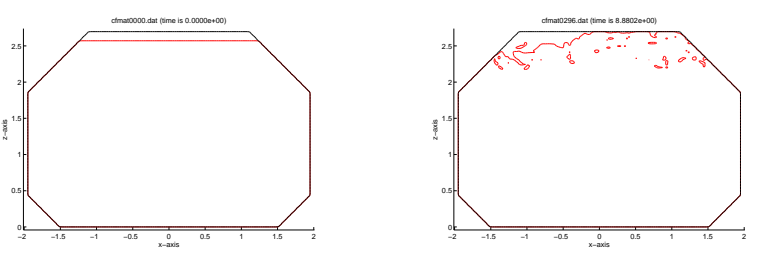

Figure 12. Snapshots of the 95 percent filling-ratio sloshing simulation. The left picture shows the fluid configuration at $t=0 \mathrm{~s}$, the right picture the configuration at $t=8.8 \mathrm{~s}$.

Regarding the development of the water height, the water height is equal to the tank height during a part of the oscillation period. As soon as the air pocket passes through the water height sensor, the water height decreases, as visible in Figure 13.

From Figure 13 it appears that the two-phase flow simulation correlates better with the experimental data than the onephase simulation. This has to do with the simulation of the complete flow domain for two-phase flow, while for one-phase flow a number of 'empty' air cells near the ceiling of the tank are not calculated.

The pressure development at the top right corner $(x=$ $1.801 m, z=2.009 m$ ) is shown in Figure 14 for the one-phase simulation, two-phase simulation and the experiment.

The pressure level development in Figure 14 shows a rather good correlation between the simulations and the experiment. The short pressure spikes for the one-phase simulation are not visible in the two-phase simulation and the experiment, these short pressure spikes are related to rapid changes in cell labeling at the boundary of the flow domain. 


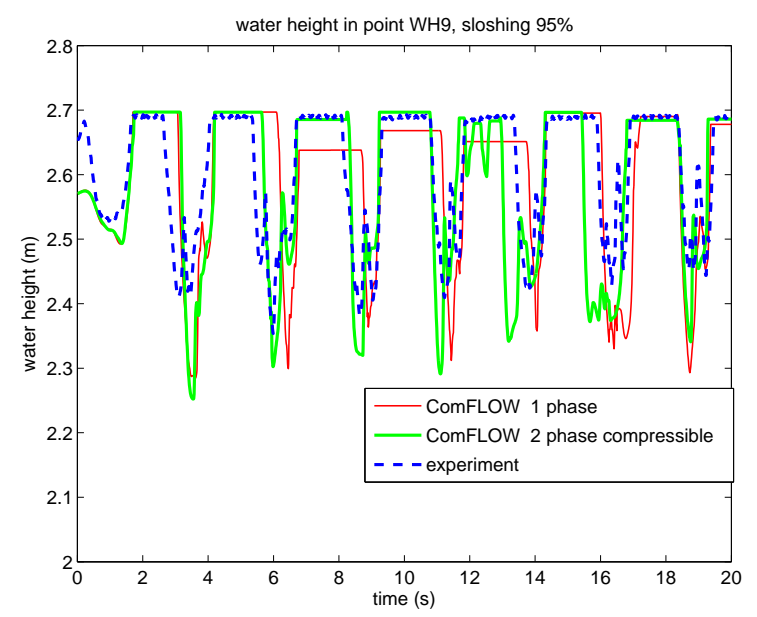

Figure 13. Water height development for 95 percent filling-ratio at sensor 9, about $1 \mathrm{~m}$ from the left side wall.

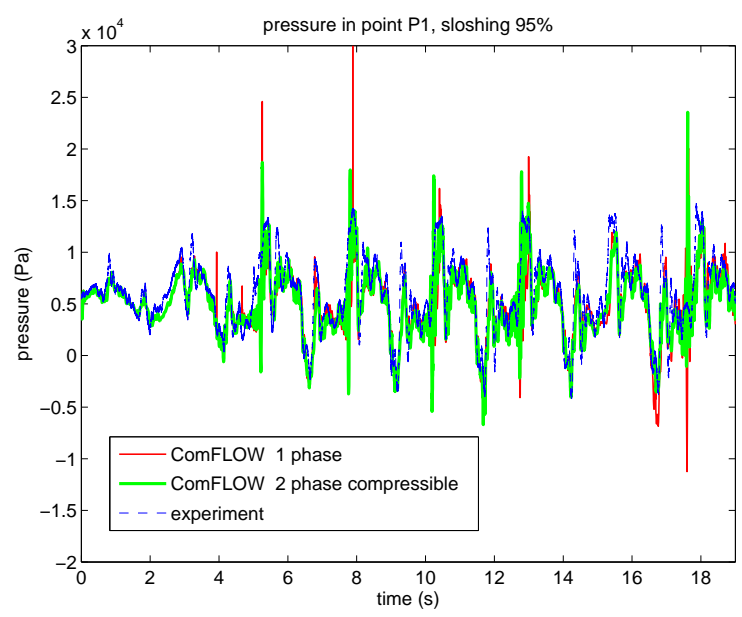

Figure 14. Pressure level development for 95 percent filling-ratio at pressure sensor 1, at the side wall.

\section{CONCLUSIONS}

The hydrodynamics of different offshore applications can be simulated numerically using an iVOF Navier-Stokes solver. This paper shows the results of the validation of the compressible twophase model on a 1:10 scale sloshing model experiment.

The main flow variables velocity, pressure and density are calculated in all grid cells. Particular attention has been paid to the calculation of the density at the free surface, as the density calculation at cell edges affects the pressure calculation. By doing this in the right way, spurious velocities are prevented.

To further improve the simulation of the dynamics of the air phase, the second phase is compressible. The air is subjected to adiabatic compression and expansion.

For different test cases, it has been shown earlier that the simulation results from the two-phase model have a better agreement with available measurement results [7] than the results from the corresponding one-phase model. Now the numerical model has been tested on more elaborate sloshing experiments on 1:10 model scale. For calm weather conditions in combination with low tank filling ratios, the results of the one-phase model already give a good prediction of water heights and pressure levels. However, for high filling ratios of the tank the results of the two-phase flow model are better, as the dynamics of the air phase play an important role in that case.

\section{ACKNOWLEDGMENT}

This research is supported by the Dutch Technology Foundation STW, applied science division of NWO and the technology programme of the Ministry of Economic Affairs in The Netherlands.

\section{REFERENCES}

[1] Greco, M., Colicchio, G., Faltinsen, O.M. Application of a 2D BEM-level set domain decomposition to the greenwater problem. 20th International Workshop on Water Waves and Floating Bodies, Longyearbyen, Norway, 2005.

[2] Gerrits, J., Veldman, A.E.P. Dynamics of liquid-filled spacecraft. Journal of Engineering Mathematics, 45:21-38, 2003.

[3] Hirt, C.W., Nichols, B.D. Volume Of Fluid (VOF) Method for the Dynamics of Free Boundaries. Journal of Computational Physics, 39:201-225, 1981.

[4] Kleefsman, K.M.T., Fekken, G., Veldman, A.E.P., Buchner, B., Iwanowski, B. A Volume-Of-Fluid Based Simulation Method For Wave Impact problems. Journal of Computational Physics, 206:363-393, 2005.

[5] Scardovelli, R., Zaleski, S. Direct Numerical Simulation of Free-Surface and Interfacial Flow. Annual Review of Fluid Mechanics, 31:567-603, 1999.

[6] Veldman, A.E.P., Gerrits, J., Luppes, R. Helder, J.A., Vreeburg, J.P.B. The numerical simulation of liquid sloshing on board spacecraft. Journal of Computational Physics, 2007, in press.

[7] Wemmenhove, R., Loots, G.E., Luppes, R., Veldman, A.E.P. Modeling two-phase flow with offshore applications. 24th Offshore Mechanics and Arctic Engineering Conference, paper 67460, Halkidiki, Greece, 2005.

[8] Yabe, T., Xiao, F., Utsumi, T. The constrained interpolation profile method for multiphase analysis. Journal of Computational Physics, 169:556-593, 2001. 